\title{
Effect of silane modification on mechanical and thermal properties of ethylene vinyl acetate (EVA)/zeolite composites
}

\author{
N. D. Zaharri, N. Othman \& Z. A. Mohd Ishak \\ School of Materials and Mineral Resources Engineering, \\ Engineering Campus, Universiti Sains Malaysia, Malaysia
}

\begin{abstract}
In this research, both untreated and silane treated zeolite filled ethylene vinyl acetate (EVA) composites were prepared in a melt-mixing process and followed by compression molded using hot press machine according to standard test specimen. For silane surface treatment, zeolite was treated with 3-aminopropyltriethoxysilane (AMPTES) prior to the mixing process. The effect of untreated and AMPTES treated zeolite loading from 5 up to 25 volume percentages on the properties of EVA/zeolite composites were evaluated. A combination of Fourier Transform Infrared Radiation (FTIR) and scanning electron microscopy (SEM) analysis were done to characterize the resultant treated zeolite. Tensile test was performed in order to study the mechanical properties of the composites. EVA filled with AMPTES treated zeolite showed better tensile properties compared to EVA filled with untreated zeolite, which might be an indication of enhanced dispersion of zeolite in the composites. Meanwhile for morphological study using SEM, the micrograph of EVA/untreated zeolite composites exhibit several voids, agglomerations and also brittle morphology. On the contrary, the micrograph of AMPTES treated zeolite filled EVA composites showed more fibrilous and ductile morphology which suggests that the silane treatment is capable of improving an interfacial adhesion between EVA matrix and zeolite. Besides, thermal properties of the composites were also characterized by using thermogravimetric analysis (TGA) and differential scanning calorimetry (DSC). The results showed that the application of the silane treatment increases both decomposition and melting temperature of EVA/zeolite composites.
\end{abstract}

Keywords: EVA, zeolites, silane treatment, tensile properties, morphology, thermal stability. 


\section{Introduction}

The application of polymers filled with inorganic component has gained considerable attention mainly because of their excellent strength-to-weight ratios. In fact, enhanced properties arise from incorporating inorganic mineral fillers into polymers, such as increased modulus, heat deflection temperature and fire retardant properties [1]. The common mineral fillers that widely used in polymer industry are calcium carbonate, talc, mica, kaolin and wollastonite. In this study, zeolite is chosen as a filler as it provides many advantages such as economical and less harmful compared to other synthetic fillers. Plus, zeolite is commercially attractive due to its unusual crystalline structures yielding unique chemical properties. Zeolite consists of a tetrahedral network of oxygen and silicon atoms where aluminium replaces some of the silicon in order to form alumino-silicates. It is also known in widespread applications such as molecular sieves, catalysis, ion exchange materials and most importantly as mineral filler in polymer matrices [2].

However, most mineral fillers including zeolite exhibit hydrophilic ionic nature, which contributes to a major problem in ensuring the hydrophobic polymer matrix to interact interfacially with the filler. Therefore, a modification method has to be performed in order to improve the adhesion and compatibility between the filler and matrix, thus obtaining composites with better properties. This is essential as the mechanical performance of polymer composites is greatly depending on the effectiveness of the bond between polymer matrix and filler in transferring stress across the interface [3].

One of the most effective coupling agent in thermoplastic composites is silane coupling agent which is usually used for treating fillers commonly silicas, clays and other silicates. This is believed to improve the filler-polymer adhesion, preferably via chemical bonds, hence improving the performance of the composite materials [4]. Liang and $\mathrm{Li}$ [5] claimed that the strong interfacial interactions between clay and polymer matrix were responsible for better dispersion of clay and improved thermal resistance of the composites in comparison with unmodified composites.

The goal to this study is to investigate the effect of silane modification on mechanical and thermal properties of zeolite/EVA composites. All the results will be discussed and compared with the untreated zeolite/EVA composites.

\section{Experimental procedure}

In this study, ethylene vinyl acetate (EVA) with specification Cosmothene EVA H2020 was used as polymer matrix. The melt flow rate and vinyl acetate content of this EVA is $1.5 \mathrm{~g} / 10 \mathrm{~min}$ and $15 \mathrm{wt}$ \%, respectively. Meanwhile, mordenite type of zeolite mineral species was used as natural filler. Zeolite is basically a hydrous alumino-silicates having density of $1.914 \mathrm{~g} / \mathrm{cm}^{3}$ with chemical formula $\left(\mathrm{Ca}, \mathrm{Mg}_{2}, \mathrm{~K}_{2}\right) \mathrm{Al}_{2} \mathrm{Si}_{10} \mathrm{O}_{24} 7 \mathrm{H}_{2} \mathrm{O}$. Prior to the compounding process, zeolite was ground into powder form using a ring mill machine. After the grinding process, 
the exact particle size was determined by Malvern Mastersizer particle size analyzer. The result obtained was $5.62 \mu \mathrm{m}$.

\subsection{Silane surface treatment}

$0.5 \mathrm{wt} \%$ AMPTES coupling agent to zeolite was hydrolyzed in the mixture of ethanol/water (90/10 vol.\%) for 15 minutes at room temperature. Then, zeolite was mixed and treated with the hydrolyzed solutions. Next, the solution was slightly stirred for 30 minutes at $50^{\circ} \mathrm{C}$. Finally, the treated zeolite was dried in an oven at $120^{\circ} \mathrm{C}$ for 24 hours.

\subsection{Compounding technique}

Prior to compounding, zeolite powder and EVA were dried in an oven at $80^{\circ} \mathrm{C}$ for 24 hours. The composites were compounded using an internal mixer Thermo Haake Polydrive with Rheomix, R600/610 model. The processing conditions were set at $130^{\circ} \mathrm{C}, 7 \mathrm{~min}$ of mixing time and $50 \mathrm{rpm}$ of rotor speed. After that the samples were compression molded for the required test pieces.

\subsection{Tensile test}

The tensile properties were determined using an electromechanical Instron machine, 3366 model. The tensile test was conducted according to ASTM D 638 with specimen dimensions of $150 \mathrm{~mm}$ x $120 \mathrm{~mm}$ x $1 \mathrm{~mm}$. The speed of the cross head was $50 \mathrm{~mm} / \mathrm{min}$ meanwhile the gauge length was $50 \mathrm{~mm}$. The tensile mechanical properties, such as tensile strength, elongation at break and Young modulus, were determined by calculating the average values of the five tested dumbbell-shaped samples

\subsection{Scanning Electron Microscopy (SEM)}

A scanning electron microscope, Leica Cambridge Ltd. S360 model was used to study the composites morphology. Samples were examined after sputter coating with gold to avoid electrostatic charging and poor image resolution.

\subsection{Thermogravimetric Analysis (TGA)}

A PerkinElmer Pyris TGA-6 thermogravimetric analyzer was used to measure the weight losses and decomposition temperature of pure EVA and its composites in the temperature range of $20-600^{\circ} \mathrm{C}$ with a heating rate of $20^{\circ} \mathrm{C} / \mathrm{min}$ and under a flow of nitrogen.

\subsection{Differential Scanning Calorimetry (DSC)}

DSC analysis was carried out using a PerkinElmer Pyris DSC-6. $5 \mathrm{mg}$ of samples were heated from 30 to $190^{\circ} \mathrm{C}$ at a rate of $10^{\circ} \mathrm{C} / \mathrm{min}$ and held at $190^{\circ} \mathrm{C}$ for 2 minutes then they were cooled to $30^{\circ} \mathrm{C}$ at $10^{\circ} \mathrm{C} / \mathrm{min}$ and reheated under the same conditions to $190^{\circ} \mathrm{C}$. The crystallinity degree for the composites is 
determined by the ratio $\Delta \mathrm{Hm} / \Delta \mathrm{Hm}_{100}$, where $\Delta \mathrm{Hm}$ is the heat of fusion in joule per gram polyethylene segments in the EVA samples meanwhile $\Delta \mathrm{Hm}_{100}$ is the heat of fusion in joule per gram pure polyethylene with $100 \%$ crystallinity. The $\Delta \mathrm{Hm}_{100}$ is 281 joule per gram pure polyethylene with $100 \%$ crystallinity [6].

\subsection{Fourier Transform Infrared Radiation (FTIR)}

FTIR, Perkin Elmer Spectrum One model machine was used to investigate the presence of functional group in EVA/zeolite composites. Besides, silane treated zeolite powder were tested to prove the effectiveness of the applied surface modifications. The range of wavelength was $550-4000 \mathrm{~cm}^{-1}$ with 4 scan times.

\section{Results and discussion}

\subsection{Tensile}

Figure 1 illustrates the effects of silane treatment on tensile strength of EVA/zeolite composites. It can be noted that at similar zeolite loading (15 vol.\%), the tensile strength of EVA/zeolite/AMPTES composites has been improved from 7.65 $\mathrm{MPa}$ to $13.97 \mathrm{MPa}$ as compared to untreated zeolite/EVA composites. This indicates that the addition of AMPTES has improved the adhesion and the interfacial strength between EVA and zeolite causing the stresses transferred from EVA to zeolite has become more effective, thus increasing the tensile strength of the composites [7]. Silane coupling agents can enhance the filler-matrix interfacial adhesion through reactions of surface

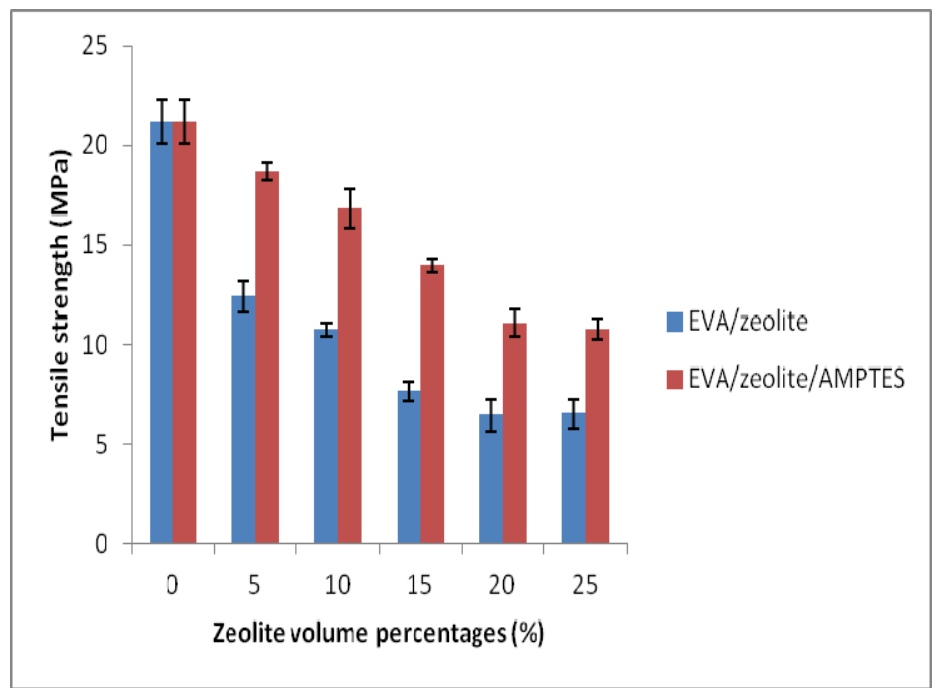

Figure 1: The effect of silane surface treatment on the tensile strength of EVA/zeolite composites. 
hydroxyl groups of filler and silanol groups of coupling agent. The induction of chemical reactions between the functional groups of the silane-modified filler and the polymer matrix further enhance the interaction between the components of the polymer composites.

From Figure 2, it shows that the elongation at break of AMPTES treated EVA/zeolite composites has significantly improved compared to zeolite filled EVA composites without silane surface treatment at similar zeolite loading. Particularly, at $15 \mathrm{vol} \% \%$ of zeolite loading, the elongation at break of EVA/zeolite composites has increased from 361\% to 597\% with the presence of AMPTES coupling agent. It is believed that the improvement in elongation at break might also be due to the lubricating effect of a coupling agent on the polymer composites. Furthermore, one may observe outstanding lubricating effect in composites, if the ratio of filler and coupling agent exceeds some critical content. The improved filler/matrix adhesion leads to higher deformability of the composites hence higher value of elongation at break [8].

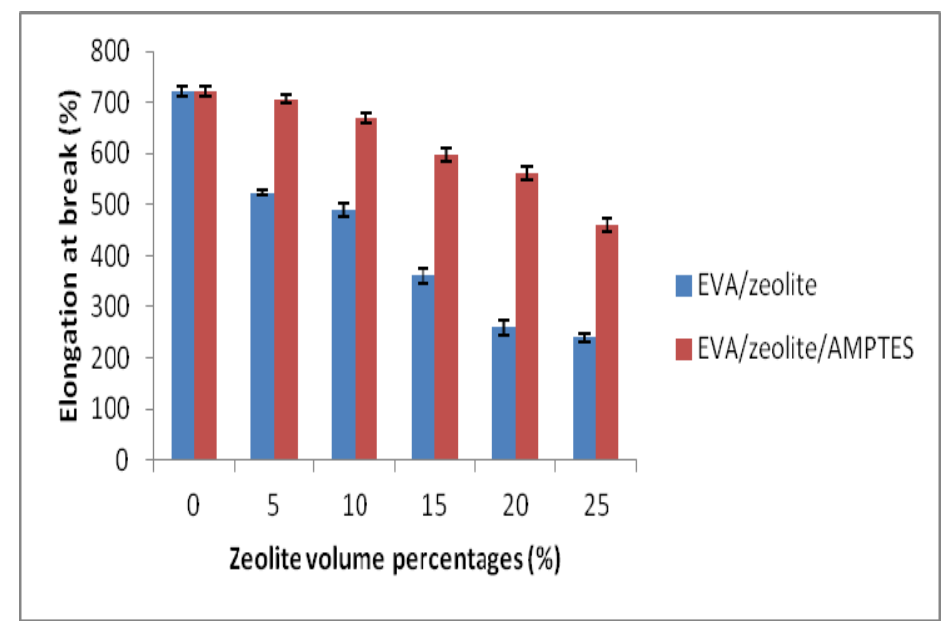

Figure 2: The effect of silane surface treatment on elongation at break of EVA/zeolite composites.

Figure 3 indicates that at similar zeolite loading (15 vol.\%), AMPTES treated EVA/zeolite composites exhibit higher tensile modulus compared to untreated one. This obviously results in an increase in the efficiency of perfect bonding between EVA matrix and zeolite, which consequently gives rise to higher tensile modulus. The enhancement in tensile modulus in the presence of AMPTES is attributed to the ability of the coupling agents to form chemical bonding between polymer matrix and filler [9]. This chemical bond may improve the interaction at interface areas between the two components more than physical bonding and van der Waals forces, hence increasing the tensile modulus of the polymer composites. 


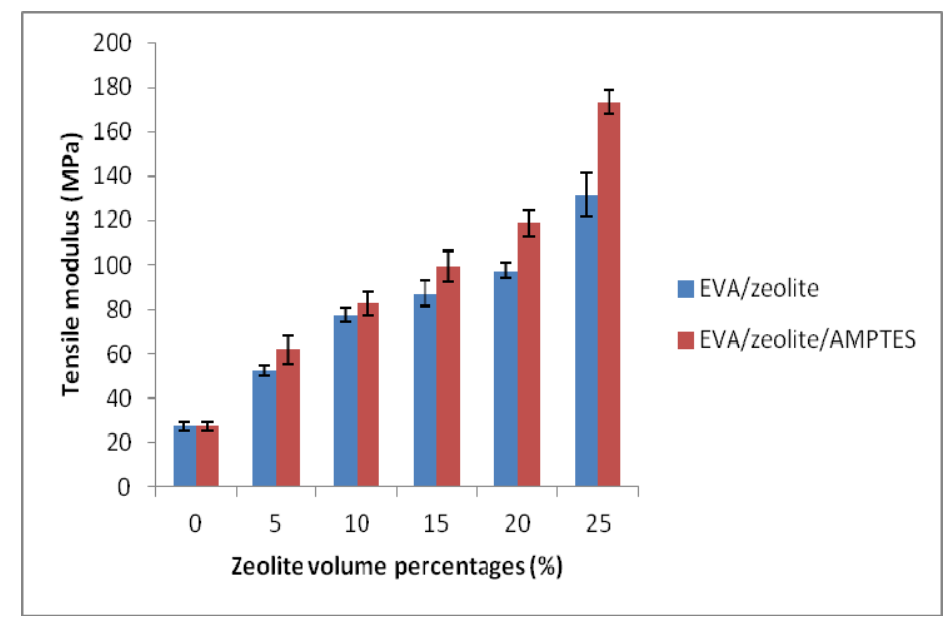

Figure 3: The effect of silane surface treatment on tensile modulus of EVA/zeolite composites.

\subsection{TGA}

The effect of silane modification on thermal degradation of EVA/zeolite composites is evident in Figures 4 and 5. The addition of AMPTES coupling agent on EVA/zeolite composites result in better thermal properties compared to EVA/zeolite composites without silane treatment. For example, the maximum

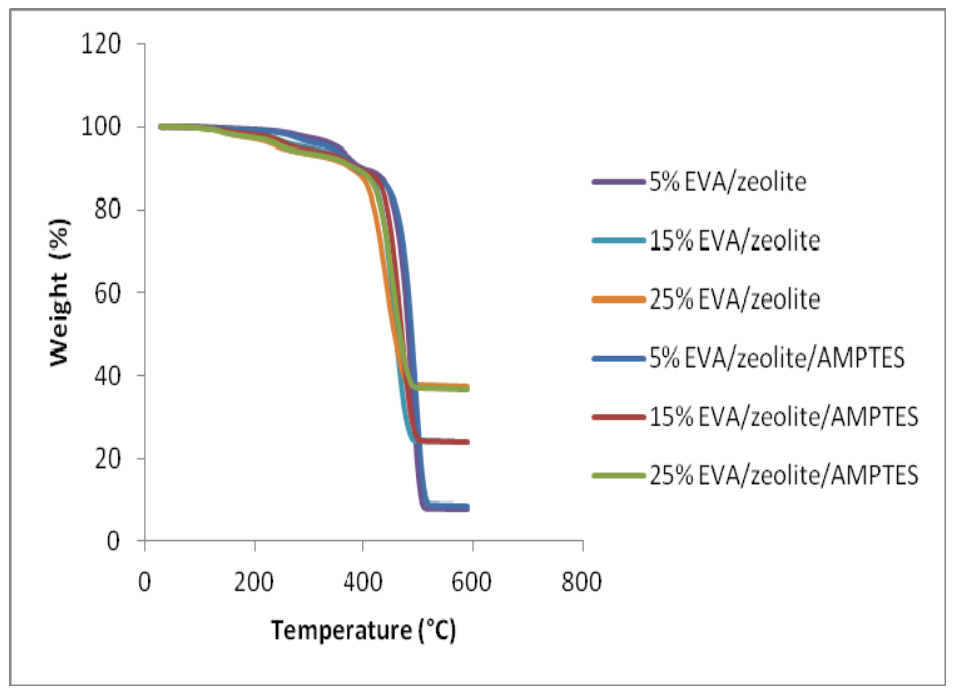

Figure 4: $\quad$ TGA curves of untreated and AMPTES treated zeolite filled EVA composites at various zeolite contents. 


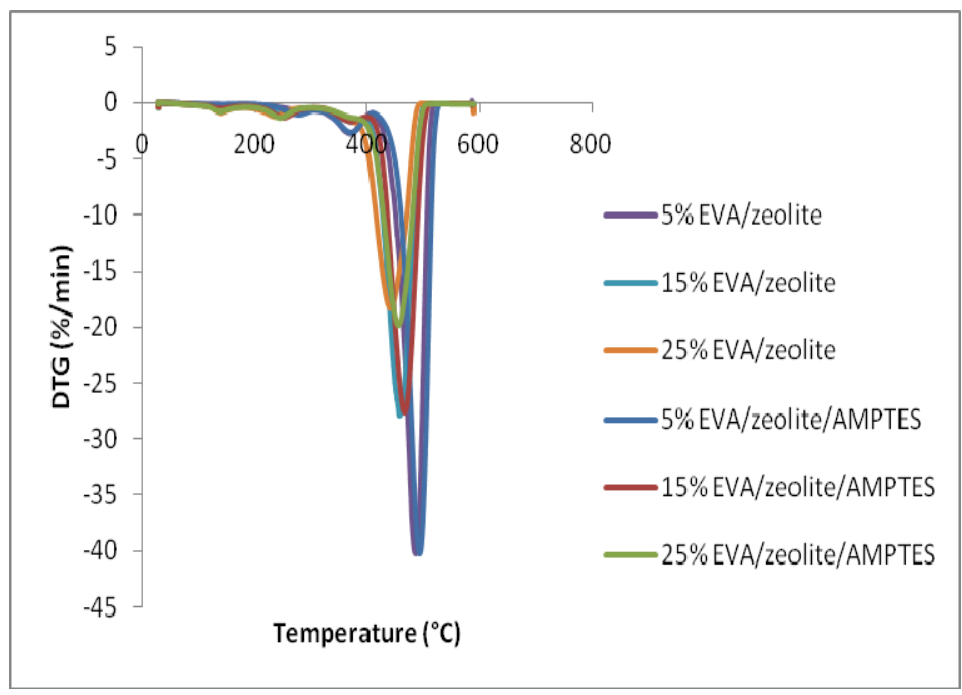

Figure 5: DTG thermograms of untreated and AMPTES treated zeolite filled EVA composites at various zeolite contents.

degradation temperature $\left(\mathrm{T}_{100}\right)$ of 15\% EVA/zeolite composites shifted from 459 to $467^{\circ} \mathrm{C}$ in the presence of AMPTES coupling agent. This is attributed to the attachment of silanol group of AMPTES on the zeolite surface, which causes the interaction adhesion between EVA and zeolite to increase, hence leads to increments in thermal stability [10].

\subsection{DSC}

The DSC thermograms of EVA/zeolite composites and EVA/zeolite/AMPTES composites at various zaolite loadings are shown in Figure 6(a) and (b), respectively. Melting temperature of EVA/zeolite composites containing AMPTES coupling agent shifts to higher temperature in comparison with unmodified EVA/zeolite composites. The modification of the filler reinforced polymer composites using modifier, compatibilizer or coupling agent was found to improve the thermal resistance of the composites due to the stronger interaction between the filler and the polymer matrix that was caused by the formation of the covalent bond at the interface [11]. It can be noted that the addition of AMPTES coupling agent gave somewhat lower crystallinity values of EVA/zeolite composites with respect to the corresponding untreated composites. This is believed due to formation of chemical bonds induced by AMPTES coupling agents, which results in strong interfacial interaction between zeolite and EVA, hence hindering the crystallization process.

\subsection{FTIR}

Figure 7 indicates the FTIR spectrums of both untreated and AMPTES-treated zeolite filled EVA composites. It can be noted that the silane functional groups 

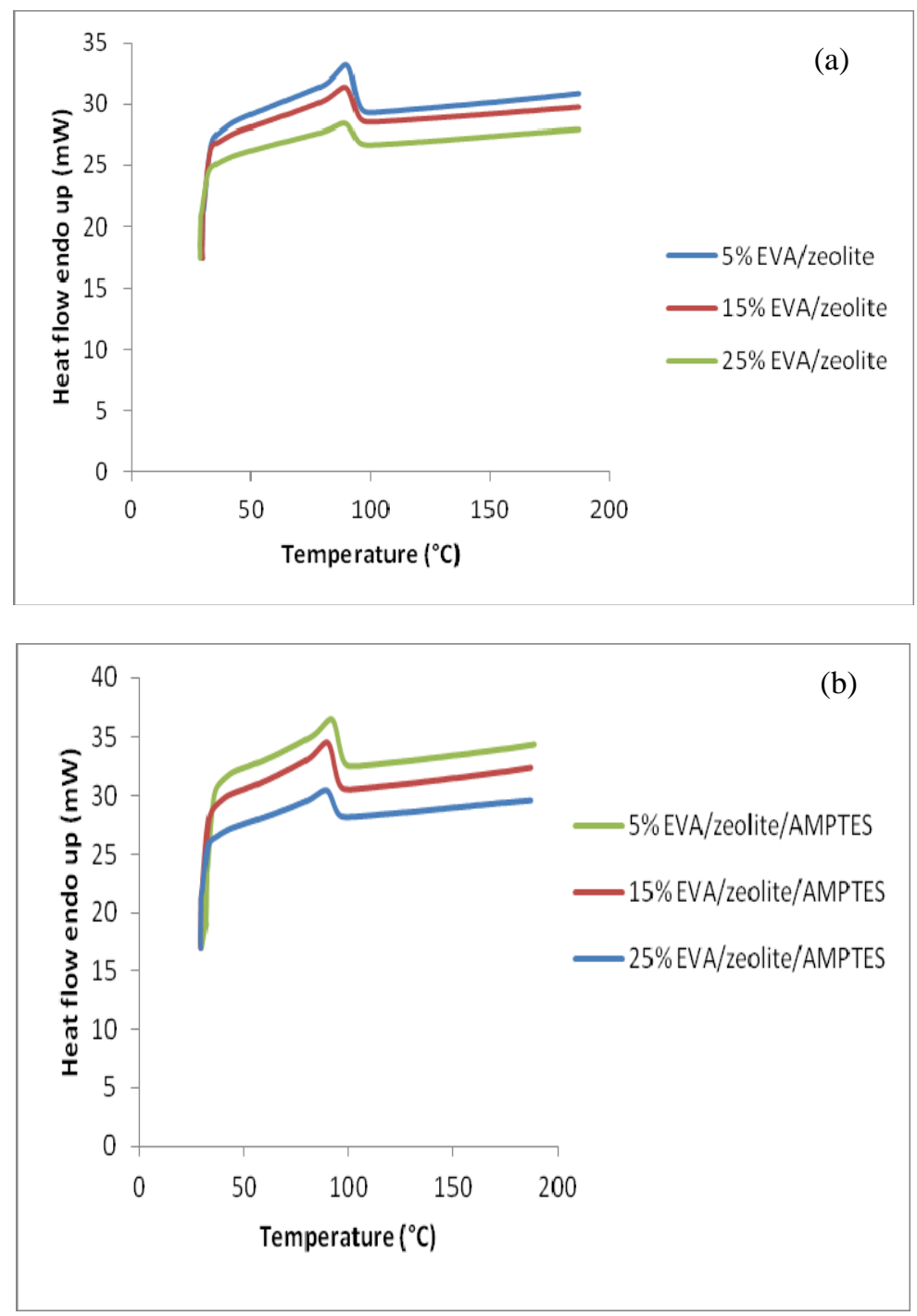

Figure 6: The heating melt DSC curves of (a) EVA/zeolite composites and (b) EVA/zeolite/AMPTES composites with different zeolite content.

are not easily detected, due to the strong and broad peaks of $\mathrm{Si}-\mathrm{O}\left(1050 \mathrm{~cm}^{-1}\right)$ and $\mathrm{OH}-\left(3445.54 \mathrm{~cm}^{-1}\right)$ presented in zeolite. However, the spectrum of AMPTES treated zeolite showed increased intensity of a peak corresponding to $\mathrm{Si}-\mathrm{O}$ stretching in comparison with that of untreated zeolite. Also, the spectrum of AMPTES treated zeolite showed a small and broad peak at $2931 \mathrm{~cm}^{-1}$ associated with the stretching of $\mathrm{CH}_{2}$ in the silane chain. The other reason 
proving the existence of AMPTES coupling agent on the zeolite surface is at $1424 \mathrm{~cm}^{-1}$, where the peak is assigned to the stretching of C-N indicating the existence of amine groups in the AMPTES treated zeolite. The presence of these new peaks suggests that the silane functional group has been successfully introduced onto the zeolite surfaces. Similar as the result obtained by organomodified zeolite, the spectrum of AMPTES-treated zeolite also exhibits a reduction in intensity at $3620 \mathrm{~cm}^{-1}$ (-OH stretching peak) indicating an increased hydrophobicity in zeolite due to the existence of an organic layer on the zeolite surface and that the bond linkage between the AMPTES surface and the zeolite surface has occurred.

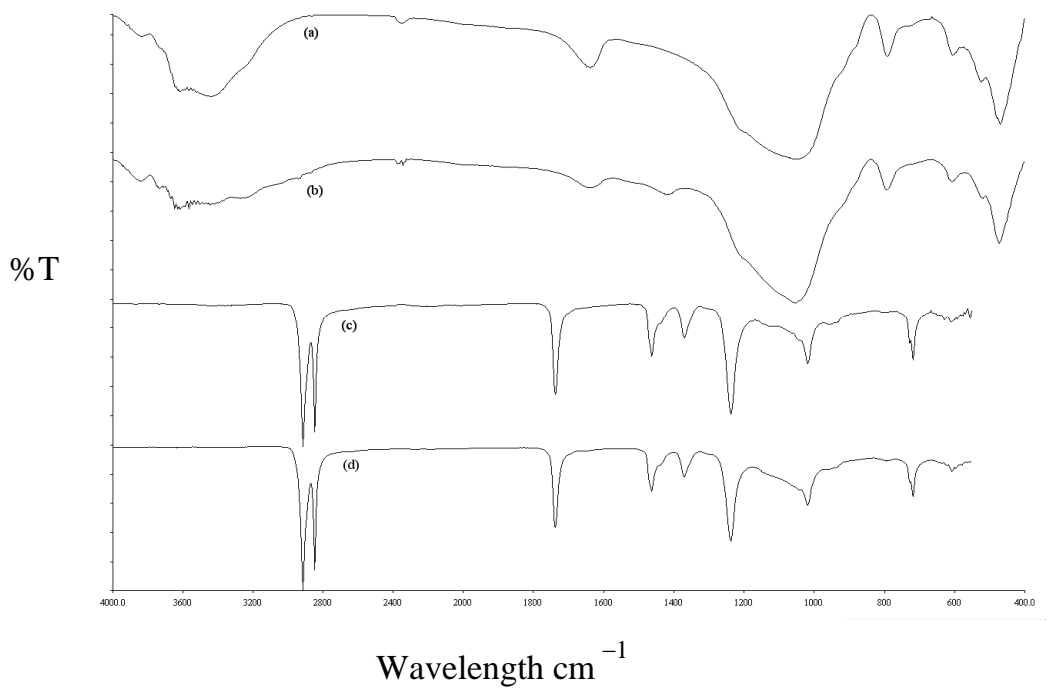

Figure 7: FTIR diagram of (a) zeolite (b) AMPTES-treated zeolite (c) EVA/zeolite composites and (d) EVA/AMPTES-treated zeolite composites.

\subsection{SEM}

Figures 8-9 show the SEM micrograph of tensile fractured surface of 5 vol.\% and 25 vol.\% of EVA/zeolite and EVA/zeolite/AMPTES composites. It can be seen clearly; more extensive fibril structure morphology with almost no voids can be observed on the fractured surface of EVA/zeolite/AMPTES composites (Fig. 9). The formation of fibrilous morphology, as can be seen in Figures 9(a) and (b) indicates a more ductile failure compared to the unmodified EVA/zeolite composites as shown in Fig. 8(a) and (b). This proves that during deformation, the AMPTES treated zeolite absorb more stress before fracture hence results in the significant improvement properties of the composites, as evident in mechanical and also thermal analysis. The enhancement of the interface can be explained by the decrease in surface energy of the filler with surface modifier, 

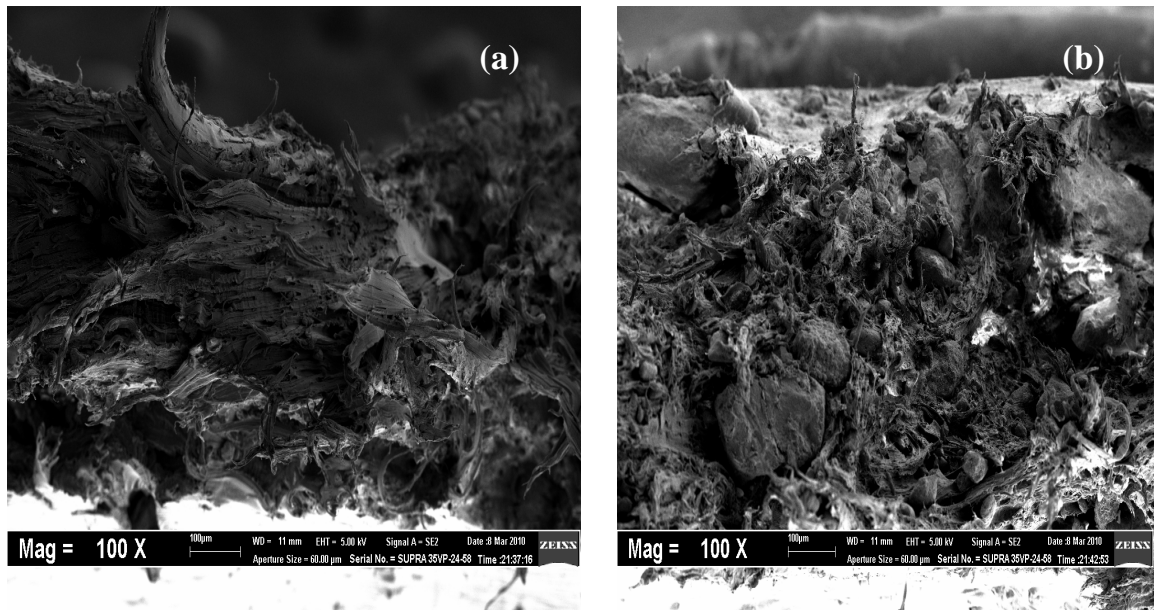

Figure 8: $\quad$ SEM micrograph of tensile fractured surface of (a) 5 vol.\% and(b) 25 vol.\% of EVA/zeolite composites.
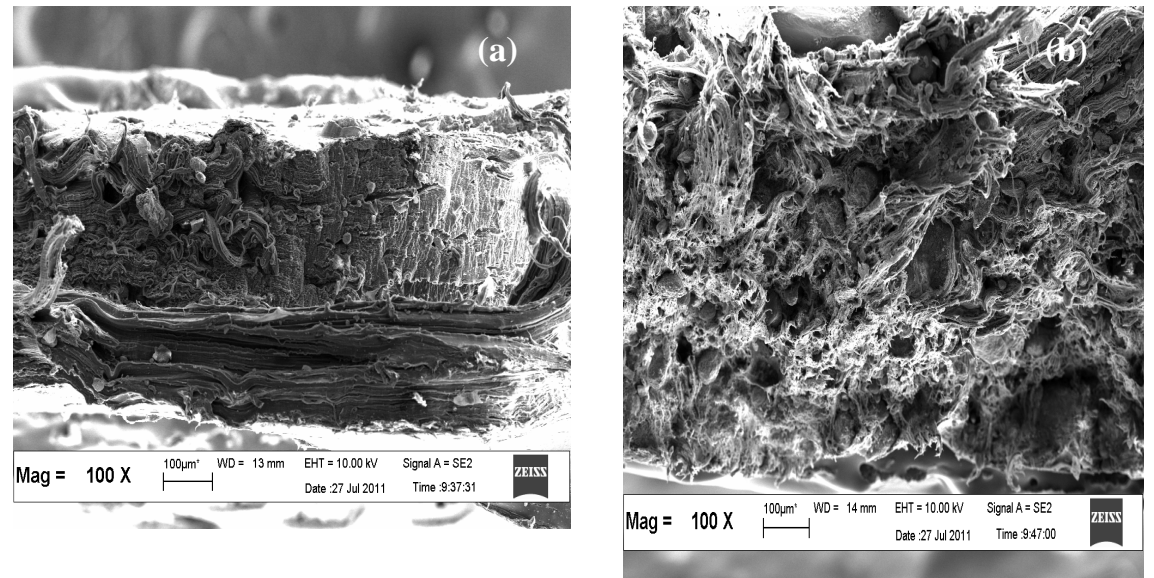

Figure 9: SEM micrograph of tensile fractured surface of (a) 5 vol.\% and (b) 25 vol.\% of EVA/zeolite/AMPTES composites.

which leads to the improvement of compatibility between the filler and polymer matrix. This suggests that the surface modifications of EVA/zeolite composites with silane treatment are capable of increasing the compatibility and interfacial adhesion between EVA matrix and zeolite particles. These observations validate the results of mechanical and thermal discussed earlier. 


\section{Conclusions}

Zeolite was successfully treated with AMPTES. Thus, the mechanical and thermal properties of zeolite/EVA/AMPTES composite are better than the untreated one. The morphological study revealed the formation of fibrillation, which corresponds to the mechanical results.

\section{References}

[1] Fu, S.Y., Xi-Qiao, F., Bern, L., Yiu-Wing, M. (2008) Effects of particle size, particle/matrix interface adhesion and particle loading on mechanical properties of particulate-polymer composites, Composites Part B: engineering, 39, p. 933-961.

[2] Domka, L. (1994) Modification estimate of kaolin, chalk, and precipitated calcium carbonate as plastomer and elostomer fillers, Colloidal Polymer Science, 272, p. 1190-202.

[3] Hsueh, C.H. (1987). Effects of aspect ratios of ellipsoidal inclusions on elastic stress transfer of ceramic composites, Composites Parts A, 72, p. 344-347.

[4] Rebeca, B., Aurora, L., Maria, J., Luis, B. (2008) Effects of vinyltrimethoxysilane on thermal properties and dynamic mechanical properties of polypropylene-wood flour composites, Journal of Applied Polymer Science, 109, p. 1197-1204.

[5] Liang, J.Z., Li, Y. (1998) Prediction of tensile yield strength of rigid inorganic particulate filled thermoplastic composites, Journal of Materials Processing Technology, 83, p. 127-130.

[6] Hassler, R., Khonakdar, H.A., Jafari, S.H (2007). Glass-TransitionTemperature Depression in Chemically Crosslinked Low-Density Polyethylene and High-Density Polyethylene and Their Blends with Ethylene Vinyl Acetate Copolymer, Journal of Applied Polymer Science, 1654-1660.

[7] Han, M.J., Byeong, C.K., Eun, H.K. (2005) Structure and properties of EVOH/organoclay nanocomposites, Journal of Materials Science, 40, p. 3783-3787.

[8] Svehlova, V., Poloucek, E. (1994) Mechanical properties of talc-filled polypropylene. Influence of filler content, filler particle size and quality of dispersion, Angew. Makromol. Chem., 214, p. 91-99.

[9] Pukanszky, B., Tudos, F. (1989) The possible mechanisms of polymerfiller interaction in polypropylene- $\mathrm{CaCO}_{3}$ composites, Journal of Materials Science Letter, 8, p. 1040-1042.

[10] Yuhai, S., Yuanfang, L., Demin, J. (2007) Preparation and Properties of Natural Rubber Nanocomposites with Solid-State Organomodified Montmorillonite, Journal of Applied Polymer Science, 107, p. 2786-2792.

[11] Doan, T.T.L., Brodowsky, H., Mader, E. (2007) Jute fibre/polypropylene composites II. Thermal hydrothermal and dynamic mechanical behavior, Composites Science and Technology, 67, p. 2707-2714. 